\title{
Development and Application of Methane Leakage Monitoring System for Gas Transmission Pipeline
}

\author{
Qijun Wang*, Shiqi Wei \\ Petroleum and Gas Engineering at Southwest Petroleum University, Chengdu 610500, Sichuan Province, China
}

*Corresponding author: Qijun Wang, xyz317@163.com

\begin{abstract}
Oil and gas pipeline transportation, as a relatively safe way of oil and gas transportation, undertakes most of the transportation tasks of crude oil and natural gas. Oil and gas pipeline accidents affect a wide range of consequences. Therefore, the oil and gas pipeline leakage detection is paid more and more attention. In this paper, ultra-low power methane gas sensor is selected to collect methane gas concentration in the air, and wireless network technology is used to build a wireless network sensor system with $4 \mathrm{G}$ function. Through the sensor distribution along the pipeline, it can intuitively and accurately judge whether there is a micro-leakage in the pipeline, and understand the diffusion situation after the leakage. The sensor system has high reliability and stability, and has high value of popularization and application.
\end{abstract}

Keywords: Oil and gas pipeline; Leak detection; Ultra-low power consumption; Methane gas sensor

Publication date: November 2021; Online publication: November 30, 2021

\section{Introduction}

With the rapid development of China's economy and society, its economic aggregate has jumped to the forefront of the world. Accordingly, the total energy consumption has also continued to grow substantially, and China has become a major energy producer and consumer ${ }^{[1]}$. The long-distance pipeline is responsible for the transportation of most crude oil and natural gas, and plays an important role in guaranteeing economic development, promoting people's livelihood, social stability and national defense construction ${ }^{[2]}$. However, the service age of the pipelines built in the 1970 s is approaching or more than 50 years, and the aging is serious. Oil and gas pipeline accidents affect a wide range of consequences. Therefore, the technology of leak detection and location of large diameter and long-distance oil and gas pipeline is paid more and more attention ${ }^{[3]}$.

Methane is the main component of natural gas. By detecting the small content of methane gas around the pipeline, the micro-leakage situation of the pipeline can be understood as soon as possible, and the huge loss caused by the aggravation and spread of leakage can be reduced. However, the conventional on-line methane detection device has high power consumption and is difficult to be used in the field environment with inconvenient power supply and limited communication conditions. Therefore, a low-power methane concentration sensor based on wireless Mesh network is designed in this paper. The ultra-low-power nondispersion-infrared (NDIR) gas sensor MIPEX-04 is used to collect methane gas concentration in the air ${ }^{[4]}$, through wireless Mesh network communication technology ${ }^{[5]}$, and finally realized the real-time online acquisition and wireless transmission of methane concentration, and the average power consumption is less than $10 \mathrm{~mW}$.

The sensor is powered by a built-in high-energy lithium battery, supplemented by green energy such as temperature difference, solar energy and vibration ${ }^{[6]}$. To achieve full life cycle power supply maintenance-free. Thus, the deficiency of existing methane concentration detection sensors can be made 
up.

\section{Design of wireless network sensor}

In order to realize the low-power methane concentration sensor with wireless Mesh network communication, two parts of the system hardware and the underlying software need to be completed. The following two parts will be described in detail.

\subsection{System hardware design}

The current of methane measurement sensors on the market is generally large, often in the power level of hundreds of $\mathrm{mW}$ to several $\mathrm{W}$, which is difficult to be used in low power consumption environment. MIPEX infrared absorption methane concentration sensor produced by OPTOSENSE Company of Russia is used in this paper ${ }^{[7]}$, its operating power consumption is less than $3 \mathrm{~mW}$, greatly reducing the power consumption of the sensor.

MIPEX infrared gas sensor adopts luminescent and photodiode based on solid alloy A2B4-A2B6, which can be used for hydrocarbon detection more efficiently, and solves the problems of high temperature influence, poor stability and large volume of traditional NDIR technology in gas measurement. The MIPEX small volume sensor uses a miniature parabolic mirror with high light transmission efficiency, and a new TYPE of LED. By optimizing the radiation spectrum and special signal processing algorithm, the sensor is highly stable in the operating temperature range. The $\mathrm{PbSe}-\mathrm{CdSe}$ photodiode, based on a solid film solution, is 10 times more sensitive than pyroelectric detectors and offers unparalleled temperature and long-term stability compared to earlier thermoelectric detectors. Figure 1. is the physical picture of MIPEX-04 series sensor.

The processor ensures the normal operation of the whole wireless sensor. Its main tasks include collecting concentration value, temperature value, battery power, wireless network networking and data transmission, and low power consumption processing. This paper uses low Power processor STM32L412 microcontroller as the system processor, which has Flex Power Control function ${ }^{[8]}$, improves the flexibility of power mode management and reduces the overall power consumption of applications. The circuit of the processor is shown in Figure 2.

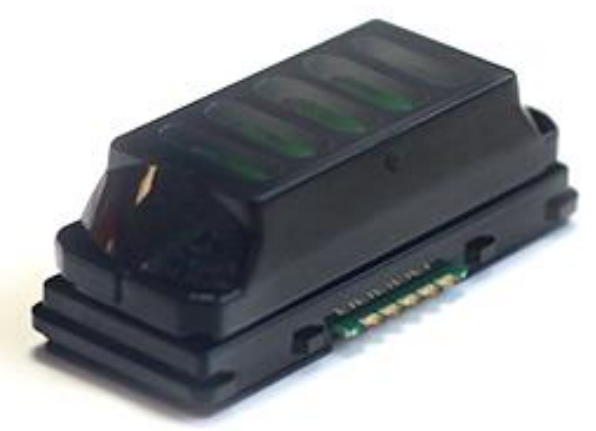

Figure 1. MIPEX-04 series sensor

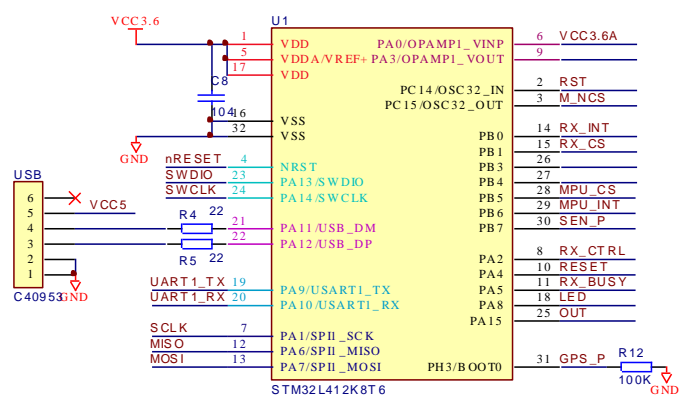

Figure 2. Main processor schematic.

Digi International Inc. (Digi) has been dedicated to the design and production of iot connected products and iot devices since the beginning of the Internet of Things. Digi International launched DigiMesh on the RF module with built-in $900 \mathrm{MHz}$ and $2.4 \mathrm{GHz}$ XBee ${ }^{[9]}$.DigiMesh is an advanced peer-to-peer protocol for battery-powered networks that simplifies Mesh networks while providing advanced features such as router hibernation and support for dense networks. DigiMesh is a peer-to-peer Mesh protocol with no parent-child structure. In this way, all sensors can be put into hibernation state to save power, and at the same time, they can be used as routers and terminal devices at the same time, ensuring communication quality and 
effectively reducing power consumption. Its communication network topology is shown in Figure 3.

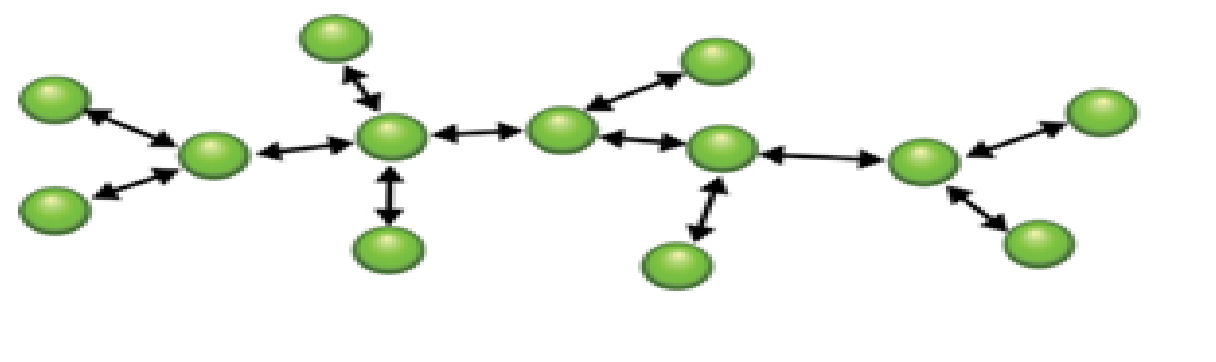

Figure 3. DigiMesh communication network topology diagram.

Through the above work, the hardware design of the sensor node is completed. Figure 4. is the physical picture and installation schematic diagram.

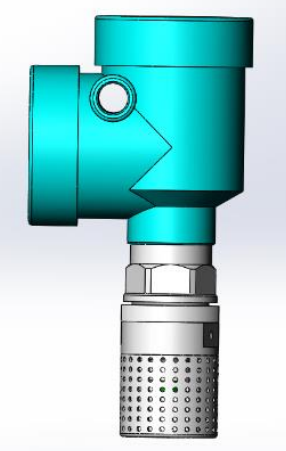

a. Physical picture of wireless network sensor

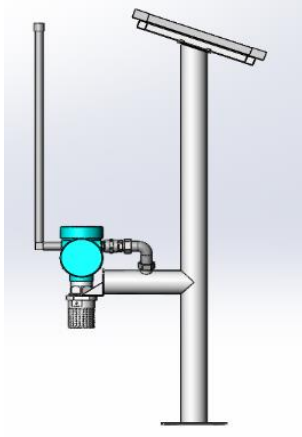

b. Installation diagram

Figure 4. Sensor physical and installation diagram.

\subsection{Underlying software design}

In order to optimize and improve the program, the underlying software of sensor node includes Bootloader program and application program, which realizes IAP function through function pointer program jump, redirection interrupt direction table and change program download address. In order to realize the Bootloader function, the ROM size of the processor is reasonably partitioned into the Flash space. The Flash partition and its related description are shown in Table 1.

Table 1. Flash partition table

\begin{tabular}{|c|c|c|c|}
\hline $\begin{array}{l}\text { Starting } \\
\text { address }\end{array}$ & $\begin{array}{l}\text { The final } \\
\text { address }\end{array}$ & The size & instructions \\
\hline 0x8000000 & 0x8003FFF & $16 \mathrm{~KB}$ & Bootloader program area, reset startup \\
\hline 0x8004000 & 0x80217FF & $118 \mathrm{~KB}$ & Application area, Bootloader jump start procedure \\
\hline 0x8021800 & 0x803EFFF & $118 \mathrm{~KB}$ & $\begin{array}{l}\text { New application area, temporarily store the new } \\
\text { firmware when upgrading }\end{array}$ \\
\hline 0x803F000 & 0x803F7FF & $2 \mathrm{~KB}$ & Firmware upgrade data, store the upgrade status \\
\hline 0x803F800 & 0x803FFFF & $2 \mathrm{~KB}$ & $\begin{array}{l}\text { Application data, which stores configureuration } \\
\text { parameters }\end{array}$ \\
\hline
\end{tabular}

Wireless sensor nodes and gateways are designed based on FreeRTOS ${ }^{[10]}$. FreeRTOS is an open source and tailorable multi-task real-time operating system. FreeRTOS has no limit on the number of system tasks 
and supports both priority scheduling algorithms and rotation scheduling algorithms.

The application program design mainly uses the task scheduling function of FreeRTOS and the lowpower Tickless mode to achieve a short-term low-power state during a variety of sensor data collection, data communication and idle tasks.

\section{Network platform design}

In order to realize real-time data monitoring and alarm, the network platform needs to acquire real-time data of each sensor node and conduct data analysis and processing. Therefore, the network platform is divided into data acquisition platform and data analysis platform. The design of these two parts is described in detail below.

\subsection{Design of data acquisition platform}

The data collection platform focuses on data collection and is composed of Server, gateway and each sensor node. It belongs to CS architecture (Client/Server). Each sensor node communicates with the Server through the gateway using HTTP protocol, and can obtain configuration information for updating and real-time uploading of field data. Its communication structure is shown in Figure 5.

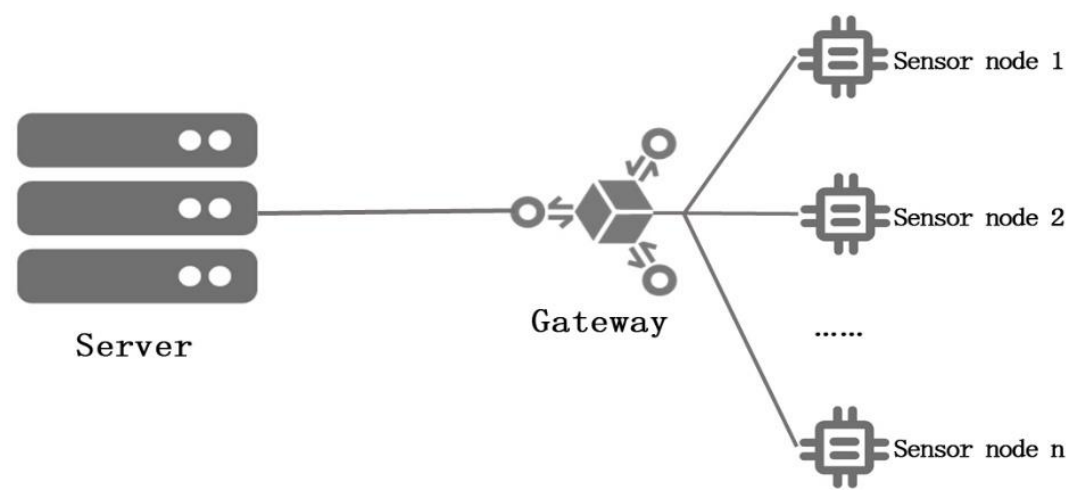

Figure 5. Network communication structure diagram.

Since the HTTP protocol is transmitted in plaintext during data transmission, there is a risk of leakage of sensitive information, so we use encryption algorithm to encrypt data before transmission. In the selection of encryption algorithm, we compare the advantages and disadvantages of DES, AES and RSA encryption algorithms and finally choose AES algorithm. Compared with DES algorithm, AES algorithm has higher security and operation speed. Compared with RSA, RSA has higher security intensity, but it also puts more pressure on the server and low-power sensors when a large amount of data is transferred.

For the convenience of data analysis platform for data analysis and processing, we store the latest data of each node in Redis ${ }^{[11]}$. In memory database to facilitate quick query, when there is a change in a certain kind of data collected by the node, the data in Redis will be updated, otherwise only the timestamp of the data will be updated; In addition, each collected node data is written to RabbitMQ ${ }^{[12]}$. In message queues, the data analysis platform processes and decides which data needs to be stored persistently.

\subsection{Data analysis platform design}

The data analysis platform analyzes and processes the collected data, and presents the analysis results. It belongs to THE BS architecture (Browser/Server). In addition, to minimize the strain on the server, we put some of the data calculation in the browser side.

To monitor whether each node is offline, we use the heartbeat mechanism for detection. Each node periodically uploads the collected data to the data collection platform. The data analysis platform analyzes 
the time of the data in the message queue. If no new data is received within the specified time, the node is considered offline. According to the corresponding algorithm to analyze the gas concentration, environmental temperature and other data, to judge whether there is abnormal environment node.

In most of the time, the data collected by a node is unchanged. If persistent storage is carried out for each node, the amount of data stored by the server will also increase sharply when the number of sensor nodes is large. Therefore, in order to reduce unnecessary data storage, we only carry out persistent storage when the data changes, and store the time when each piece of data is received. In this way, it is easy to infer what kind of data is collected during this period from the time difference between the two pieces of data, which can greatly reduce the amount of data to be stored persistently.

\section{Field application}

Through a long time of field application, it can be seen from the monitoring data of the network server that the system has strong real-time performance, stable operation and high reliability. Figure 6. and Figure 7. show the methane gas concentration data curve and temperature data curve recorded by server monitoring respectively.

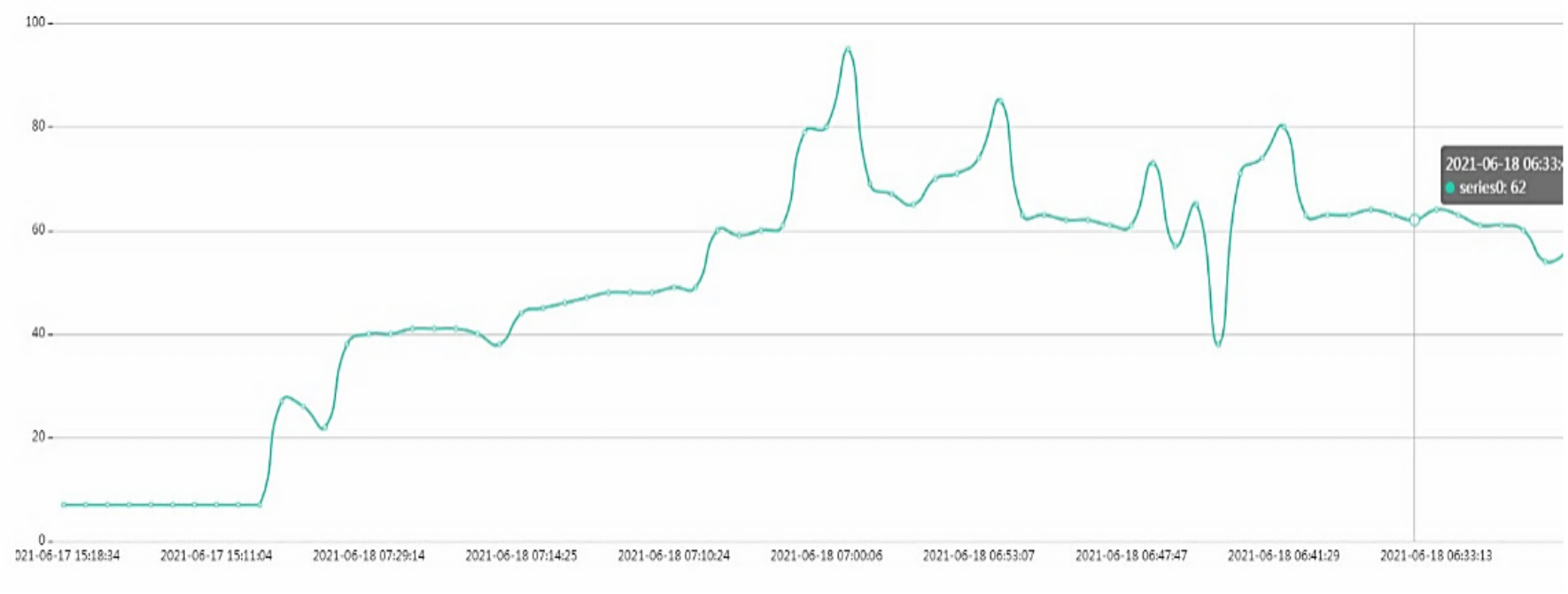

Figure 6. Monitor the methane gas concentration data curve.

Figure 7. Monitoring temperature data curve. 


\section{Conclusion}

In order to make up for the disadvantages of conventional methane gas concentration monitoring instruments, such as large power consumption, inconvenient layout and poor communication, this paper designs a methane concentration sensor that can use independent power supply in the field environment. The sensor realizes real-time monitoring and transmission of methane concentration under low power consumption based on wireless Mesh network. The long-term application in the field shows that the sensor has the advantages of low power consumption, stable operation, simple structure and convenient installation. It can not only be used in gas pipeline leakage monitoring, but also in oil and gas, chemical industry, coal mine and other high-risk environments. It has great market application and promotion value.

\section{Funding}

The 2019 Ministry of Education industry-university cooperation collaborative education project "Research on the Construction of Economics and Management Professional Data Analysis Laboratory" (Project number: 201902077020)

\section{Disclosure statement}

The author declares no conflict of interest.

\section{References}

[1] County R, 2008, Natural Gas Exploration and Development in China will have Great Development. Sinopec News, 2008.08.19.

[2] Cao S, 2014, Science and Technology Guarantee Energy Safety Transportation. General Machinery, 2014(3): 2-2.

[3] Wang J, 2010, Who Guards 70,000 km Oil and Gas Pipelines. Safety and Health, 2010(19): 38-39.

[4] Yin J, Xu Z, 2013, Design of a Wireless Gas Sensor Node with Low Power Consumption. Microcontroller and Embedded System Application, 2013(8): 21-24.

[5] Ruan X, Mao Y, 2010, IEEE Transactions on Wireless Networks, 12(1): 1-12.

[6] Wang Q, Guo Z, Peng B, 2011, Application of Energy Collection Power Supply in Petroleum Industry. Instrument User, 2011(18): 62-64.

[7] Didier R, 2015, Increasing Validity by Recombining Existing Indices: MIPEX as a Measure of Citizenship Models. Social Science Quarterly, 2015(96): 629-638.

[8] STMicroelectronics, 2017, STM32L4 Summary of Ultra-Low Power Consumption Function AN4621 Application Notes, August 2017.

[9] Ekanayake JC, Hedley CB, 2018, New Zealand. Advances in Information Provision from Wireless Sensor Networks for Irrigated Crops. Wireless Sensor Network, 2018(4): 71-92.

[10] Zhang CH, WANG YF, 2014, Transplantation and Application of FreeRTOS on STM32F103VCT6. Industrial Control Computer, 2014(12): 22-24.

[11] Redis. http://www.redis.cn/.

[12] RabbitMQ. https://www.rabbitmq.com/. 